\title{
Does Your Core Rate Structure Really Matter?
}

Jerry Hunter

University of Wisconsin - Madison, Madison, Wisconsin, United States

The Wisconsin Centers for Nanoscale Technology (WCNT) are the University of Wisconsin-Madison, College of Engineering, shared user facilities. The centers are operated as a shared use facility, where effectively all use is performed by the users. Staff are present to train users and keep the instrumentation functional, but generally do not operate instruments for users. This model is quite common in university shared facilities due to requiring fewer staff vs. a service facility where staff perform the work for users. Together, these core facilities provide equipment, facilities, and expertise in microelectronics, nano-fabrication technology, electron microscopy, micro-analysis and soft materials characterization in support of the University's research endeavor.

In this paper we will use 12.5 years of data gathered from the WCNT shared use facilities to investigate the impact of rate changes on recharge and user behavior. The dataset covers $>500,000$ sessions from 2300 users, 500 Principal Investigators, > 2000 sponsored projects and 860,000 usage hours. Core facilities like this are a key component of supporting the research enterprise of research universities. These facilities give access to expertise intensive and expensive instrumentation and allow for the sharing of costs related to these instruments among a large group of users. Even though core facilities are such a vital part of the research enterprise, most of them do not generate enough user recharge to be fully selfsufficient (reference), requiring institutional support funds to make up for operating shortfalls. There is constant pressure on core facilities to increase recharge to make up for these operating shortfalls. The most commonly proposed method to increase user recharge is to simply increase the rate paid by the users since it is a parameter over which the core facility has control. This paper will investigate the impact of increasing rates on the subsequent recharge generated in a center, in addition to other factors, such as user behavior, that the rate structure can control. 